\title{
E. coli 4.5S RNA Is Part of a Ribonucleoprotein Particle That Has Properties Related to Signal Recognition Particle
}

\author{
Véronique Ribes, Karin Römisch, Angelika Giner, \\ Bernhard Dobberstein, and David Tollervey \\ European Molecular Biology Laboratory \\ Meyerhofstrasse 1 \\ Postfach 102209 \\ D6900 Heidelberg \\ Federal Republic of Germany
}

\section{Summary}

E. coli 4.5S RNA and P48 have been shown to be homologous to SRP7S RNA and SRP54, respectively. Here we report that expression of human SRP7S in E. coli can suppress the lethality caused by depletion of 4.5S RNA. In E. coli, both RNAs are associated with P48. In vitro, both E. coli P48 and SRP54 specifically bind to 4.5S RNA. Strains depleted of 4.5S RNA strongly accumulate pre- $\beta$-lactamase and fail to accumulate maltose binding protein. These effects commence well before any growth defect is observed and are suppressed by expression of human SRP7S. Strains overproducing P48 also accumulate pre- $\beta$-lactamase. 4.5S RNA and P48 are components of a ribonucleoprotein particle that we propose to be required for the secretion of some proteins.

\section{Introduction}

Protein translocation across membranes is a basic cellular process common to all organisms. Many studies show that at least the initial stages of protein secretion in prokaryotes and eukaryotes have features in common. From both, most secreted proteins are synthesized as larger preproteins containing an amino-terminal signal sequence, which is cleaved following membrane translocation. Signal sequences are structurally and functionally conserved from prokaryotes to higher eukaryotes, as the signal sequence from one system can be recognized by the other (for review see von Heijne, 1985). Additional information within the mature domain of the protein is required to stop transfer through the membrane, in the case of some integral membrane proteins (von Heijne, 1985). Finally, in both systems, protein secretion requires a complex machinery (for review see Pugsley, 1988).

In higher eukaryotes the factor that interacts with the signal sequence prior to translocation is the signal recognition particle or SRP, a cytoplasmic ribonucleoprotein particle consisting of one RNA molecule (7SL RNA or SRP7S) of $\sim 300$ nucleotides and six distinct proteins of 9 , $14,19,54,68$, and $72 \mathrm{kd}$ (Walter and Blobel, 1982; Walter and Lingappa, 1986; for review see Siegel and Walter, 1988). The secondary structure of SRP7S has been determined; the $5^{\prime}$ region forms two small stem-loop structures, and the major $5^{\prime}$ and $3^{\prime}$ regions form a long central stem ending in two large stem-loop structures, which we will refer to as stem-loop 1 (nucleotides 124-170) and stem- loop 2 (nucleotides 176-220) (Zwieb, 1985). In vitro, SAP binds to the signal sequence as it emerges from the ribosome, imposing a complete or partial block on translation of the nascent polypeptide chain (for review see Siegel and Walter, 1988). When the SRP/ribosome complex binds to the docking protein (Meyer et al., 1982) or SRP receptor (Gilmore et al., 1982), a protein complex associated with the membrane of the endoplasmic reticulum, translation resumes and the protein is translocated across the membrane (Meyer et al., 1982).

In Escherichia coli, protein export requires both soluble and membrane peripheral and integral factors. However, while the eukaryotic components have been identified by biochemical fractionation, those of $\mathrm{E}$. coli have been identified largely by genetic techniques. These have led to the identification of five genes, secA, secB, secD, secE, and secy (Oliver and Beckwith, 1981; Kumamoto and Beckwith, 1983; Shiba et al., 1984; Gardel et al., 1987; Riggs et al., 1988; Shatz et al., 1989), that are likely to play important roles in protein secretion. Of these, the products $\operatorname{Sec} A, \operatorname{SecE}$, and SecY are associated with the membrane; their relationship to the eukaryotic docking protein is at present unclear. On the basis of suppressor analyses of signal sequence mutations, evidence of a direct interaction with the signal sequence has been presented for the SecA protein (Fikes and Bassford, 1989; Stader ot al., 1989).

Many recent studies demonstrate that in order to be translocated across the $E$. coli membrane, preproteins must have an export-competent (probably partially unfolded) conformation (Randall et al., 1987; Meyer, 1988). To maintain its translocation competence following transla. tion, the preprotein interacts with cytosolic proteins until binding to the membrane receptor for translocation. Biochemical and genetic data suggest that different preproteins can associate with different cytosolic factors, some of these being interchangeable; induction of heat shock can bypass the requirement for SecB, and SRP can replace trigger factor for in vitro translocation of OmpA (Kumamoto, 1989; Crooke et al., 1988). In the case of pre$\beta$-lactamase, the completed preprotein has been shown to interact with GroEL/GroES, while maltose binding protein (MBP) interacts with SecB (Kusukawa et al., 1989; Kumamoto and Beckwith, 1985; Bochkareva et al., 1988; Collier et al., 1988). It remains controversial whether any of the cytoplasmic factors of $E$. coli can be considered to be a direct analog of SRP that binds to the signal sequence (Lee and Beckwith, 1986; Collier et al., 1988; Crooke et al., 1988; Sanz and Meyer, 1988; Kumamoto, 1989; Watanabe and Blobel, 1989).

SRP7S-like RNAS have been identified from a wide range of eukaryotes, including animals (Gundelfinger et al., 1984), plants (Campos et al., 1988), and fungi (Brennwald et al., 1988; Poritz et al., 1988a; Ribes et al., 1988). More unexpectedly, an RNA with clear homology to eukaryotic SRP7S has also been identified in Halobacterium halobium, suggesting that archaebacteria may also con- 


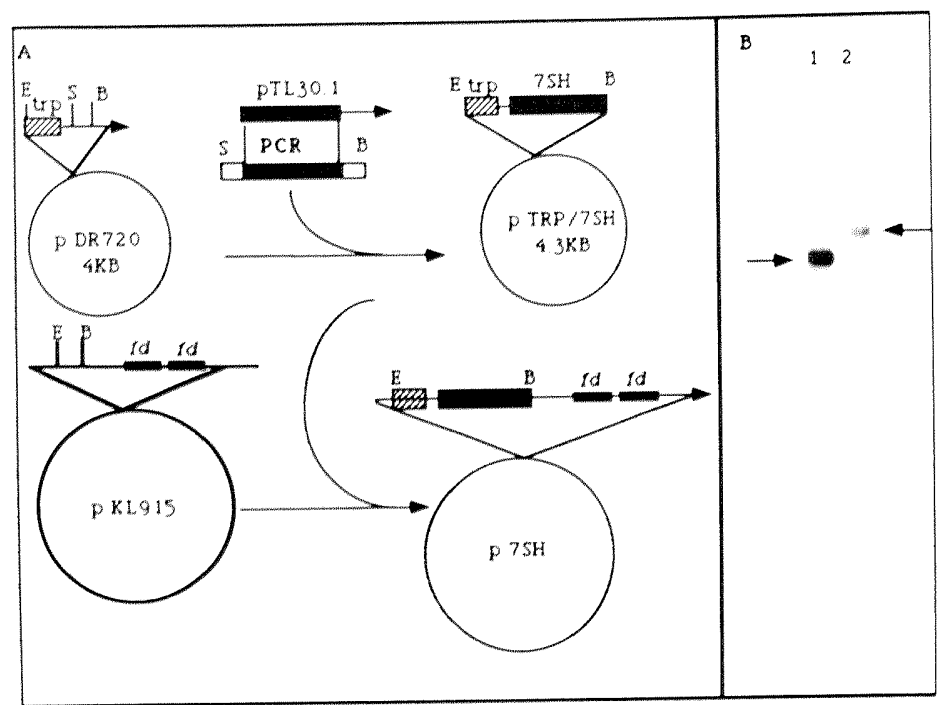

Figure 1. Construction of an Expression Vector for Human SRP7S in E. coli

(A) The human SRP7S RNA coding region was amplified from plasmid PTL30.1 (Ullu and Weiner, 1984) using PCR with oligonucleotides that include Sall and BamHI sites immediately adjacent to the $5^{\prime}$ and $3^{\prime}$ ends of the coding region, respectively. The PCR fragment obtained was subcloned into pGEM $3 Z$ and sequenced to ensure that no errors had occurred during amplification. The SRP7S coding region was recloned into the $E$. coli expression vector pDR720 (Russell and Bennett, 1982), which contains the Irp promoter. The EcoRI-BamHI fragment carrying the Trp::SRP7S fusion was recloned into pKL915, which carries the phage $f D$ transcription terminator (Sugimoto et al., 1977; Stanley and Luzio, 1984), to give plasmid p7SH.

(B) Northern hybridization of RNA extracted from HeLa cells (lane 1) or from an E. coli strain containing p7SH (lane 2). The filter was hybridized with the cloned human SRP7S gene. tain an SRP-like complex (Moritz and Goebel, 1985; Kaine, 1990). In eubacteria, small RNA species have been identified that contain a domain highly homologous to the stem-loop 2 region of SRP7S (Poritz et al., 1988b; Struck et al., 1988). In E. coli, this species is represented by the 4.5S RNA (Hsu et al., 1984), which, like SRP7S, is essential for viability (Brown and Fournier, 1984; Brennwald et al., 1988; Ribes et al., 1988).

One of the SRP proteins, SRP54, is homologous over its entire length to an $E$. coli protein of $48 \mathrm{kd}$ ( $P 48$ or $\mathrm{fh}$ ), suggesting that they may be structural homologs (Bernstein et al., 1989; Römisch et al., 1989). Proteins homologous to SRP5 4 have also been reported in the yeasts Saccharomyces cerevisiae and Schizosaccharomyces pombe (Hann et al., 1989). We show here that S. pombe SRP54 is associated with SRP7S in vivo.

The presence of two potential homologs of vertebrate SRP components in $E$. coli prompted us to determine whether these are associated in an SRP-like particle in this organism.

\section{Results}

\section{Human SRPTS RNA Can Functionally Replace}

4.5S RNA in E. coli

It has recently been shown that $4.5 \mathrm{~S}$ RNA from E. coli can be functionally replaced by RNAs from a range of other eubacteria (Brown et al., 1989; Struck et al., 1990); so, as an initial step, we determined whether 4.5S RNA can be replaced by human SRP7S.

The SRP7S coding region was amplified using the polymerase chain reaction (PCR) with oligonucleotides that insert restriction sites immediately adjacent to the ends of the coding region. The PCR fragment obtained was cloned between a Trp promoter and the phage $f D$ transcription terminator to give plasmid p7SH (see Figure 1). The Trp promoter gives strong, partially regulated tran- scription initiating 19 nucleotides $5^{\prime}$ of the mature SRP7S (Russell and Bennett, 1982). The $f D$ terminator terminates transcription at a single site and, moreover, creates a stable stem-loop structure at the $3^{\prime}$ end of the transcript (Sugimoto et al., 1977), which we expected to stabilize the RNA. In E. coli, the vector synthesizes a single abundant transcript of the expected length containing the human SRP7S sequence (Figure 1B, lane 2). As judged by staining with ethidium bromide, the abundance of the SRP7S transcript in $\mathrm{E}$. coli is around $5 \%$ of that of rRNA and similar to the abundance of 4.5S RNA.

To determine whether the SRP7S transcript can functionally replace $4.5 \mathrm{~S}$ RNA, we used a strain in which $4.5 \mathrm{~S}$ RNA synthesis is under the control of a Tac promoter (Ptac) and the endogenous 4.5S RNA gene (ffs) has been disrupted (Tac::ffs strain) (Brown and Fournier, 1984; Bourgaize and Fournier, 1987). Removal of the inducer, IPTG or lactose, results in the loss of 4.5S RNA synthesis, with growth arrest and cell death after 4-5 generations (Bourgaize and Fournier, 1987; Figure 2A). In strains also expressing a basal level of the SRP7S transcript, the growth arrest is delayed. Induction of high level expression of the Trp promoter allows wild-type growth (Figure $2 A)$. The same growth curve was obtained for seven independent transformants carrying $\mathrm{p} 7 \mathrm{SH}$, but not with transformants carrying the same vector without the human SRP7S sequence. At the time points indicated (1-5 in Figure $2 A$ ) cells were removed from the cultures, and RNA and protein were prepared. Northern analysis of the RNA (Figure 2B) shows that in Tac::ffs strains the level of 4.5S RNA quickly falls to a very low level in the absence of induction (Figure 2B, ff(-IPTG)). Previous studies show that $\sim 30 \%$ of the wild-type level of $4.5 \mathrm{~S}$ RNA is required to support growth (Bourgaize and Fournier, 1987), and the continued growth of the strain carrying p7SH (Figure 2B, $\mathrm{ff}+\mathrm{p} 7 \mathrm{SH}$ ) must be due to the presence of the SRP7S transcript. On prolonged culture ( $>10$ generations) the growth 


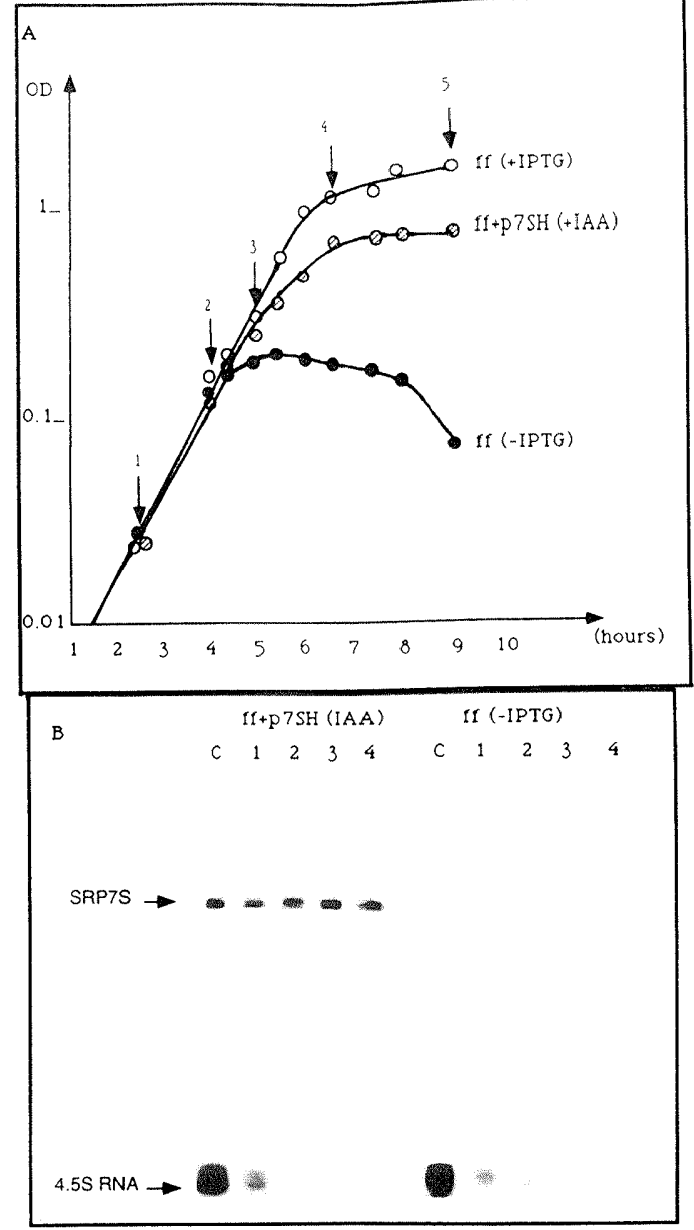

Figure 2. Growth Curves of Tac::ffs Strains and Northern Analysis of 4.5S Depletion

(A) The growth curves obtained for the Tac::ffs strain grown in the presence (ff(+IPTG)) or absence (ff(-IPTG)) of IPTG or for the same strain also carrying P7SH in the absence of IPTG and induced with IAA $(f f+p 7 S H(+\mid A A))$ are shown. Time points at which samples were withdrawn for viable counts and RNA and protein analyses are indicated as 1-5. The results shown are those obtained in an individual experiment; a large number of repetitions gave very similar results.

(B) RNA extracted from cells withdrawn at time points 1-4 of the growth curves shown in (A) as separated on a polyacrylamide gel and transferred for Northern hybridization. The left panel shows a Tac::fts strain harboring $\mathrm{p7SH}$ growing in the absence of IPTG and in the presence of IAA. The right panel shows a Tac::ffs strain growing in the absence of IPTG. Lane C, RNA from the corresponding strain in the presence of IPTG; lanes 1-4, RNA extracted at time points 1-4, respectively. The filter was hybridized successively for $4.5 \mathrm{~S}$ and human SRP7S, the positions of which are indicated.

of Tac::ffs strains complemented by $\mathrm{p} 7 \mathrm{SH}$ falls to about $30 \%$ of strains grown with IPTG, indicating that SRP7S is not fully capable of replacing $4.5 S$ RNA.

\section{The E. coli P48 Protein and SRP54 Can Bind} to 4.5S RNA In Vitro

As the mammalian SRP7S can functionally replace $4.5 \mathrm{~S}$ RNA, we tested whether SRP proteins (SRP19 and
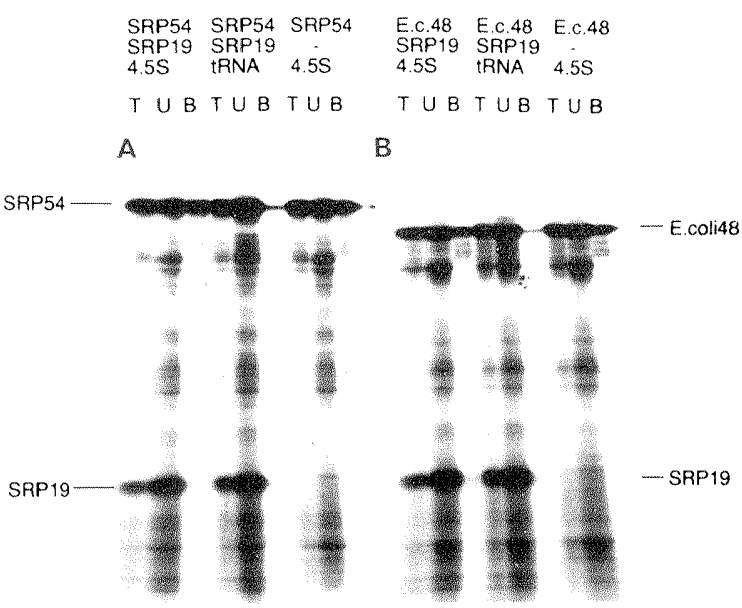

Figure 3. In Vitro Binding of E. coli P48 Protein and SRP54 to $4.5 \mathrm{~S}$ RNA

SRP54 (A) and P48 (E.coli48) (B) were incubated with 4.5S RNA in the presence or absence of SRP19. Assembled RNP was isolated by DEAE chromatography. tRNA was used instead of 4.5S RNA as negative control. An aliquot $(20 \%)$ of the total incubation mixture $(T)$, material that bound to DEAE (B), and material not bound to DEAE (unbound, U) were analyzed by SDS-PAGE and fluorography.

SRP54) are capable of interacting with 4.5S RNA. We and others have shown previously that SRP19 can directly bind to SRP7S, while SRP54 requires SRP19 for binding (Walter and Blobel, 1983; Lingelbach et al., 1988; Römisch et al., 1989). Purified 4.5S RNA was incubated in different combinations with in vitro synthesized SRP19, SRP54, and P48 proteins. Assembled RNP was isolated by binding to DEAE-Sepharose, and proteins were recovered from the bound and unbound fractions by TCA precipitation and separated on an SDS-polyacrylamide gel (Figure 3). Comparison of total (Figure 3, T lanes), unbound ( $U$ lanes), and bound ( $B$ lanes) fractions shows that SRP54 (Figure 3A) and P48 (E.coli48, Figure 3B) bind to 4.5S RNA. SRP19 (Figure 3A) does not detectably bind to 4.5 S RA, and surprisingly, the binding of SRP5 4 is not dependent on the presence of SRP19.

\subsection{S RNA and SRP7S Are Associated with the E. coli P48 Protein In Vivo}

As an initial experiment to determine whether SRP7S is associated with $\mathrm{E}$. coli proteins in vivo, we compared the sucrose gradient mobility of the RNA extracted under native conditions and following phenol extraction (Figure 4). A cell lysate was prepared from an $E$. coli strain expressing SRP7S, under nondenaturing conditions. Half of the material was extracted with phenol at $4^{\circ} \mathrm{C}$, and both fractions were loaded onto identical 5\%-20\% sucrose gradients. Following centrifugation, RNA was extracted from sucrose gradient fractions, separated on a polyacrylamide gel, and transferred for Northern hybridization. Figure 4 shows the position of SRP7S from native (N) (upper panel) and phenol-extracted $(\varphi)$ (lower panel) material. In the phenol-extracted material, the peak of SRP7S is 


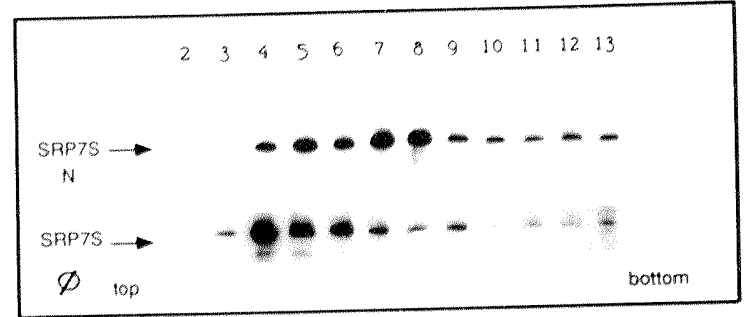

Figure 4. In Vivo Association between Human SRP7S and E. coli Proteins

Sucrose gradient separation of a lysate of $E$. coli prepared under native conditions (N) (upper panel) or following phenol extraction at $4^{\circ} \mathrm{C}(\varphi)$ (lower panel). After centrifugation. ANA was extracted, separated on a polyacrylamide gel, and transterred for Northern hybridization. The distribution of SRP7S is shown.

clearly displaced toward the top of the gradient, strongly indicating an association with $\mathrm{E}$. coli proteins in vivo. The peak of SRP7S is at a position similar to that of SRP from $S$. pombe run on a parallel gradient, indicating a sedimentation of around 10S (Poritz et al., 1988a). The peak of ribosome-associated SRP7S (see below) is not seen because ribosomes are pelleted under the conditions used.
To determine whether P48 is one of the protein species associated with SRP7S and 4.5S RNA in vivo, we raised antibodies against a synthetic peptide present in the SRP54 protein (amino acids 103-117) in a region highly conserved between SRP54 and P48 and predicted to be on the outside of the native structure. One of the sera produced (anti-SRP54/P48 antiserum $730=$ Anti $48 \mathrm{~K}$ in figures) was shown to cross-react with the E. coli P48 protein and to strongly and specifically immunoprecipitate in vivo labeled $\mathrm{P} 48$, under native and denatured conditions (Figure 5A, P48). To demonstrate its specificity, the antiserum was used to decorate Western blots of total soluble protein from $E$. coli. The reaction with P48 from wild-type (Figure 5A, lane 2) or P48-overexpressing (Figure 5A, lane 4) strains is specifically blocked by addition of the peptide against which the serum was raised (Figure 5A, lanes 3 and 5).

To demonstrate that the serum recognizes an evolutionarily conserved epitope, it was used to immunoprecipitate SRP54 from lysates of S. pombe (Figure 5C). Coprecipitated RNAs were recovered, separated on a polyacrylamide gel, and transferred for Northern hybridization. Figure $5 \mathrm{C}$ shows that $\mathrm{S}$. pombe SRP7S is coprecipitated by the anti-SRP54/P48 antiserum 730 (Anti-48K lane) but not
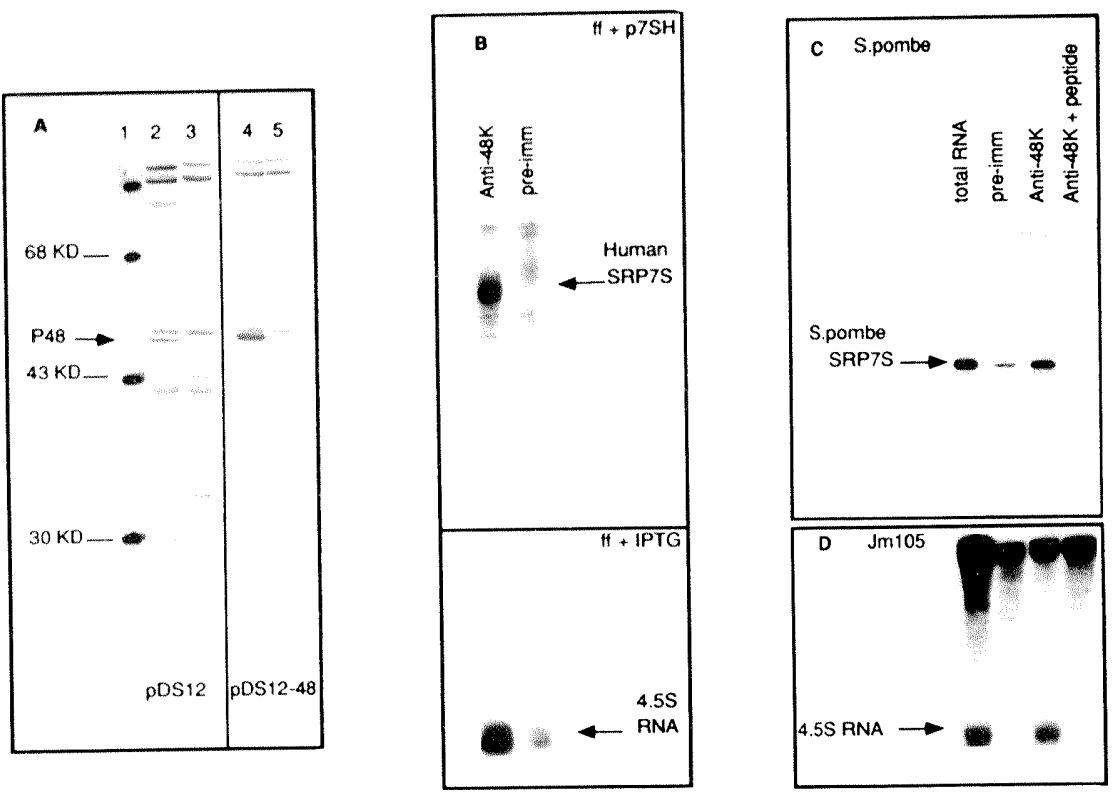

Figure 5. Immunoprecipitation of SRP7S and 4.5S RNA with the Anti-SRP54/P48 Serum 730

(A) Western blot of total soluble protein from strains of E. coli carrying the control plasmid (pDS12) or P48 overexpression vector (pDS12-48) decorated with anti-SRP54/P48 serum 730 in the absence or presence of the competitor peptide. Lane 1, molecular marker; lanes 2 and 4 , reaction of serum 730 with P48; lanes 3 and 5 , reaction of serum 730 blocked by addition of the peptide against which the serum was raised.

(B) Immunoprecipitation from lysates of E. coli expressing human SRP7S transcript (upper figure) or 4.5S RNA synthesized from the Tac::ffs construct (lower figure). RNA recovered following immunoprecipitation using the anti-SRP54/P48 antiserum 730 (Anti-48K lane) or the preimmune serum (preimm lane) was separated by polyacrylamide gel electrophoresis and analyzed by Northern hybridization.

(C) Immunoprecipitation from a lysate of S. pombe. Total RNA from a cell lysate equivalent to that used for immunoprecipitation (total RNA lane) and RNA recovered following immunoprecipitation using preimmune serum (pre-imm lane), antiserum 730 (Anti-48K lane), or antiserum 730 plus

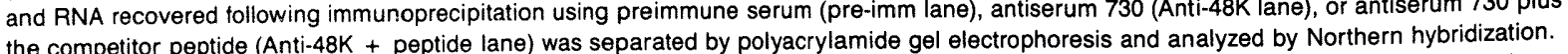
(D) Immunoprecipitation from a lysate of a wild-type strain of E. coli (Jm105). Procedures are the same as in (C). The upper bands are the large IRNA species, which were cohybridized as a control for RNA recovery.

Coprecipitated RNAs were recovered, separated on a polyacrylamide gel, and transferred for Northern hybridization. The positions of the SRP7S

(C) and 4.5S RNA (D) transcripts are shown. 


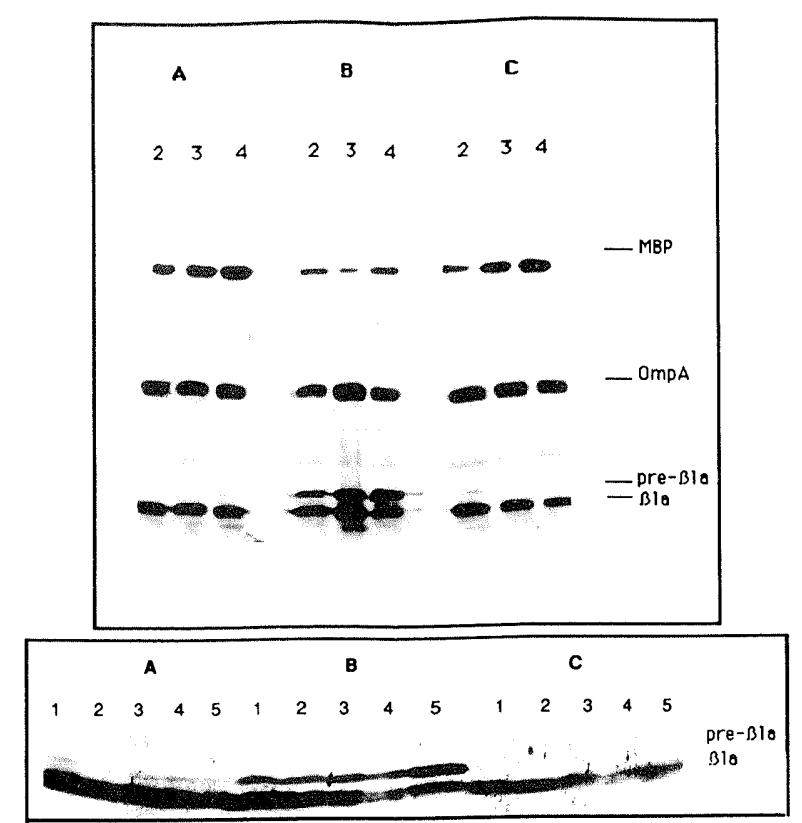

Figure 6. Processing of Presecreted Proteins in Strains Lacking 4.5S RNA

Proteins extracted from cells withdrawn at time points 1-5 of the growth curves shown in Figure $2 \mathrm{~A}$ were separated by polyacrylamide gel electrophoresis and transferred for Western blotting. A Tac::ffs strain induced for 4.5S RNA synthesis (A); a Tac::ffs strain in the absence of IPTG (B); a Tac::ffs strain harboring p7SH and growing in the absence of IPTG (C). The filter was decorated with anti-MBP, anti-OmpA, and anti- $\beta$-lactamase, and the bands were visualized using ${ }^{35} \mathrm{~S}$-labeled protein $A$ and autoradiography (anti-MPB, anti-OmpA) or goat antirabbit antibodies coupled to AP (anti- $\beta$-lactamase). For $\beta$-lactamase, two independent experiments are shown: the upper Westerns show the effects on $\beta$-lactamase, OmpA, and MBP from a single experiment; the lower panel shows the effects on $\beta$-lactamase at earlier and later time points.

by the preimmune serum (pre-imm lane) or by the serum specifically blocked for the recognition of P48 by addition of the peptide against which the serum was raised (Anti$48 \mathrm{~K}+$ peptide lane). Antiserum 730 was then used to immunoprecipitate P48 from a wild-type strain of $E$. coli (Jm105). As shown in Figure 5D, 4.5S RNA is coprecipitated with P48 (Anti-48K lane), and this immunoprecipitation is absent when the preimmune serum is used (preimm lane) or when the activity against P48 is specifically blocked by the addition of the peptide (Anti-48K + peptide lane). To show the level of nonspecific recovery, the filter shown in Figure 5D was also hybridized for the large rRNA species. The SRP7S transcript synthesized in E. coli and the 4.5S RNA transcribed from the pTac::ffs promoter construct are also coprecipitated with $\mathrm{P} 48$ by antiserum 730 (Figure 5B, Anti-48K lane) but not by the preimmune serum (Figure $5 B$, pre-imm lane).

A fraction of 4.5S RNA has been reported to be associated with ribosomes. As judged by sucrose gradient centrifugation, in our strains $\sim 5 \%$ of $4.5 S$ RNA is bound to ribosomes during growth with IPTG. The same proportion of SRP7S RNA is also associated with the ribosomes (data not shown).

\section{Strains Depleted of 4.5S RNA or Overexpressing P48 Accumulate a Presecretory Protein}

Mammalian SRP is known to be required for targetting of nascent presecretory proteins to the membrane of the endoplasmic reticulum. After having demonstrated a structural homology to part of SRP, we asked whether the 4.5S/P48 RNP is involved in protein targetting to the membrane in $\mathrm{E}$. coli. Proteins recovered from cells taken from the time course of 4.5S RNA depletion shown in Figure 2A were analyzed on Western blots. For each time point Western blots were decorated with antibodies directed against $\beta$-lactamase, MBP, OmpA, and alkaline phosphatase (AP). In strains not induced for 4.5S RNA synthesis (Figure 6B), a strong accumulation of pre- $\beta$-lactamase is observed. This accumulation is already very marked after $2.5 \mathrm{hr}$ (Figure $6 \mathrm{~B}$, lane 1, lower figure). At this time the level of $4.5 \mathrm{~S}$ RNA has already been greatly depleted, but no growth defect is yet observed (compare time points in Figures 2 and 6 ). Accumulation of pre- $\beta$-lactamase is not observed in the same strain induced for 4.5S RNA synthesis (Figure 6A) or in a strain also expressing SRP7S (Figure 6C). At late time points in Figure $6 \mathrm{~B}$ and in several other experiments we also observe a reduction of mature $\beta$-lactamase. To follow the synthesis of MBP, strains were induced with maltose at the time of transfer to medium with or without IPTG. No accumulation of pre-MBP was observed in strains depleted of 4.5S RNA, however, the level of mature MBP is greatly reduced in these strains. This impairment of MBP synthesis is suppressed in strains expressing SRP7S. In strains not induced with maltose, MBP is expressed at a low basal level. Following withdrawal of IPTG, the basal level of MBP in Tac::ffs strains becomes undetectable (data not shown). No accumulation of pre-AP is seen in strains depleted of 4.5S RNA. A slight decrease in the level of mature AP is observed at the last time point; whether this is a specific effect or due to the impaired growth at this time is not clear (data not shown). No change in the level of OmpA or pre-OmpA was observed.

When we attempted to overexpress P48 or SRP54 from plasmids carrying an inducible promoter, we observed a negative effect on cell growth. To test the effects on presecretory proteins, MC 4100 cells carrying the pDS1248 plasmid or the control plasmid pDS12 were induced with IPTG for $1 \mathrm{hr}$ or $2 \mathrm{hr}$ and labeled with [ ${ }^{35}$ S]methionine. P48 induction was monitored by immunoprecipitation with the anti-SRP54/P48 antiserum 730, which recognizes P48. In addition, OmpA and $\beta$-lactamase were immunoprecipitated and analyzed by SDS-PAGE and fluorography. The level of P48 is close to the limits of detection in cells with the control pDS12 plasmid (Figure 7A). A low level of P48 was detected in cells carrying PDS12-48 plasmid, and this is drastically increased after IPTG induction (Figure 7A). OmpA expression does not change when $\mathrm{P} 48$ is induced, and no OmpA precursor accumulation can be observed (Figure 7B). The $\beta$-lactamase gene is present on the pDS12 plasmid, and induction of the highly active $T 5$ promoter leads to overexpression of $\beta$-lactamase, presumably owing to incomplete transcription termination. This does not affect processing of pre- $\beta$-lactamase in strains carrying pDS12; however, strains carrying 


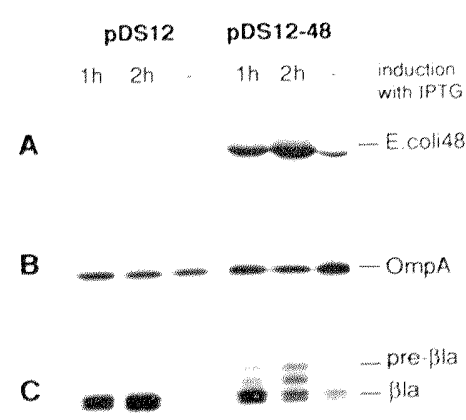

Figure 7. Processing of Presecreted Proteins in P48-Overexpressing Cells

MC4100 cells carrying either pDS12 or pDS12-48 for P48 overexpression were grown with IPTG for 1 and 2 hr or without induction and labeled with [35s 3 methionine for $10 \mathrm{~min}$. Proteins were extracted, and P48. OMPA and B-lactamase were immunoprecipitated and analyzed by SDS-PAGE and fluorography. The differences in the levels of plactamase between the induced and noninduced strains is due to the $\beta$-lactamase between the induced and noninduced strains is due to the highly active $\mathrm{T} 5$ promoter. The mature and pre- $\beta$-lactamase ran as doublets on the gel shown; this is seen for both the pDS12 and pDS12. 48 strains, and we have occasionally seen the same effect in other experiments.

pDS12-48 accumulate pre- $\beta$-lactamase after induction of $\mathrm{P} 48$, and the level of mature $\beta$-lactamase drops (Figure $7 C)$. In strains induced with maltose, the level of accumulation of mature MBP is slightly reduced in strains overexpressing $\mathrm{P} 48$; no precursor accumulation is observed (data not shown). Overexpression of other proteins, dihydrofolate reductase or chloramphenicol acetyltransferase, did not lead to accumulation of pre- $\beta$-lactamase (data not shown).

In strains either overexpressing P48 or depleted of $4.5 \mathrm{~S}$ RNA, a marked morphological change was observed. The cells become highly elongated as is shown by comparison of strains carrying pDS12 or pDS12-48. Cell elongation, accumulation of pre- $\beta$-lactamase, and loss of mature MBP is also observed in $\operatorname{Sec} A^{t s}$ mutants.

\section{Discussion}

\section{E. coli P48 and SRP54 Bind to 4.5S RNA}

4.5S RNA and the P48 protein of E. coli are homologous to components of eukaryotic SRP, SRP7S RNA and SRP54, respectively. In E. coli, immunoprecipitation data show that 4.5S RNA and P48 are associated in a ribonucleoprotein complex. Further evidence for this association is provided by an in vitro binding assay demonstrating that under stringent conditions both P48 and SRP54 can bind 4.5S RNA.

Previous studies have shown that SRP19 can directly bind to SRP7S, whereas SRP54 cannot, and binds to the particle only in the presence of SRP19. However, SRP19 does not interact with SRP54 in the absence of SRP7S. It was thought that SRP54 binds to the particle by protein-protein interactions (Siegel and Walter, 1988), since
SRP19 alone and SRP19 + SRP54 give similar nuclease protection of stem-loops 1 and 2 . However, the binding of SRP54 to 4.5S RNA in the absence of SRP19 shows that in fact, SRP54 has direct RNA binding activity. As $4.5 \mathrm{~S}$ RNA is homologous to the stem-loop 2 region of SRP7S, this structure may include the SRP5 4 binding site. The clearest difference between 4.5S RNA and SRP7S is the presence of stem-loop 1 in SRP7S. The lack of binding of SRP19 to 4.5S RNA might suggest that SRP19 binds primarily to stem-loop 1 in SRP7S. The existence of two different conformations of SRP7S has previously been proposed (Zwieb and Ullu, 1986), involving interactions between stem-loops 1 and 2 and the central rod. Binding of SRP19 to SRP7S might induce a conformational switch, thereby opening the structure of the RNA and allowing access of SRP54 to its binding site. We are testing this model using SRP7S genes deleted for stem-loops 1 and 2 .

The number of additional proteins associated with $4.5 \mathrm{~S}$ RNA is unclear at present. It will be particularly interesting to determine whether they correspond to proteins previously identified by Sec mutations or are homologous to other SRP proteins.

Human SRP7S Can Functionally Replace 4.5S RNA We were able to obtain expression of the SRP7S sequence as a single stable transcript in E. coli. In strains in which 4.5S RNA synthesis has been placed under the control of an inducible Tac promoter, removal of the inducer results in a cessation of growth after $\sim 4.5 \mathrm{hr}$, followed by cell death and lysis (Brown and Fournier, 1984; Bourgaize and Fournier, 1987). This lethality is suppressed in cells also expressing the SRP7S transcript. Expression of SRP7S is at a level close to that of endogenous 4.5S RNA, and expression of other RNAs does not have the same effect. The suppression is therefore unlikely to be due to some general effect on RNA metabolism. Since, like 4.5S RNA, SRP7S binds to P48 in E. coli, it is likely that the SRP7S/P48 complex can directly replace the 4.5S/P48 RNP.

In E. coli, P48 binds to SRP7S, but in vitro, SRP54 requires SRP19 for binding to SRP7S. P48 in E. coli may bind to SRP7S cotranslationally, before the RNA has adopted the mature secondary structure, or the structures of the RNA and/or protein differ in vivo and in vitro. As noted above, the structure of SRP7S has been proposed to undergo dynamic changes, and the conformation in vivo might well be different from that in the high salt ( 500 $\mathrm{mM}$ ) buffer used for the in vitro binding assay.

\section{Function of 4.5S RNA}

Genetic and biochemical studies have shown that $4.5 \mathrm{~S}$ RNA interacts with the ribosome (Bourgaize and Fournier, 1987; Brown, 1987, 1989). The clearest evidence comes from the characterization of suppressor mutations in elongation factor $G$ (EF-G). These mutations reduce the level of 4.5S RNA required to support growth, apparently by greatly increasing the proportion of 4.5S RNA bound to ribosomes (Brown, 1987). Moreover, treatment with puromycin, which releases the nascent polypeptide with- 
out removing ribosomes from polysomes, also causes the release of 4.5S RNA (Brown, 1989), indicating that $4.5 \mathrm{~S}$ RNA interacts directly or indirectly with the nascent chain.

Several models have been proposed for the function of 4.5S RNA. These, however, have been based on the assumption that the RNA, rather than the 4.5S/P48 RNP complex, is the functional unit. Roles in polypeptide chain initiation (Bourgaize and Fournier, 1987) and elongation (Brown, 1989) have been proposed for 4.5S RNA. Of these, only a role in elongation is consistent with the suppressor mutations and the effects of puromycin treatment. However, a general role in the elongation of all proteins is difficult to reconcile with the low abundance of 4.5S RNA compared with ribosomes or protein elongation factors. Moreover, cell lysates from strains depleted of 4.5S RNA are reported to be competent for polypeptide chain elongation in vitro on poly $(U)$ templates (Bourgaize and Fournier, 1987). Recent in vivo labeling experiments show that even at late time points (6.8 hr after removal of inducer) 4.5S RNA-depleted strains retain substantial translational capacity, and the two-dimensional gel pattern of the major proteins is unaltered (Bourgaize et al., 1990). It is also notable that depletion of 4.5S RNA not only arrests growth, but results in rapid cell death and lysis (Bourgaize and Fournier, 1987). Cell lysis would not be expected to result from translational inhibition, but is caused by factors that interfere with outer membrane synthesis and might occur if the 4.5S RNA were required for the production of one or more essential membrane components. Together these results indicate that the site of action of 4.5S RNA is on the ribosome during polypeptide elongation. Depletion experiments, however, show that the RNA is not required for general protein synthesis, suggesting it plays a role only in the synthesis of a subset of proteins.

The earliest effect of 4.5S RNA depletion detected to date is the accumulation of pre- $\beta$-lactamase. Strong accumulation is already apparent $2.5 \mathrm{hr}$ after removal of the inducer for 4.5S RNA synthesis and is also observed within 30 min of induction of P48 overexpression. This is unlikely to be a nonspecific effect of the impaired growth on secretion since other secreted proteins (OmpA and AP) are not affected, and marked accumulation of pre- $\beta$ lactamase is observed at least 3 generation times before any detectable growth defect. It has recently been reported that depletion of 4.5S RNA results in the induction of heat shock proteins $3.5 \mathrm{hr}$ after removal of the inducer but is not detectable at $2.5 \mathrm{hr}$ (Bourgaize et al., 1990). The synthesis of heat shock proteins has been shown to be induced by the presence of denatured or incorrectly folded proteins (Goff and Goldberg, 1985), and an accumulation of cytosolic pre- $\beta$-lactamase would be predicted to elicit such a response.

\section{A Hypothetical Model for the Function of $4.5 S / P 48$ RNP in Protein Secretion in E. coli}

The following conclusions can be drawn from the above results: First, 4.5S RNA interacts with P48 and this RNP can be functionally replaced by an SRP7S/P48 complex, suggesting that the $4.5 \mathrm{~S} / \mathrm{P} 48$ RNP may be functionally

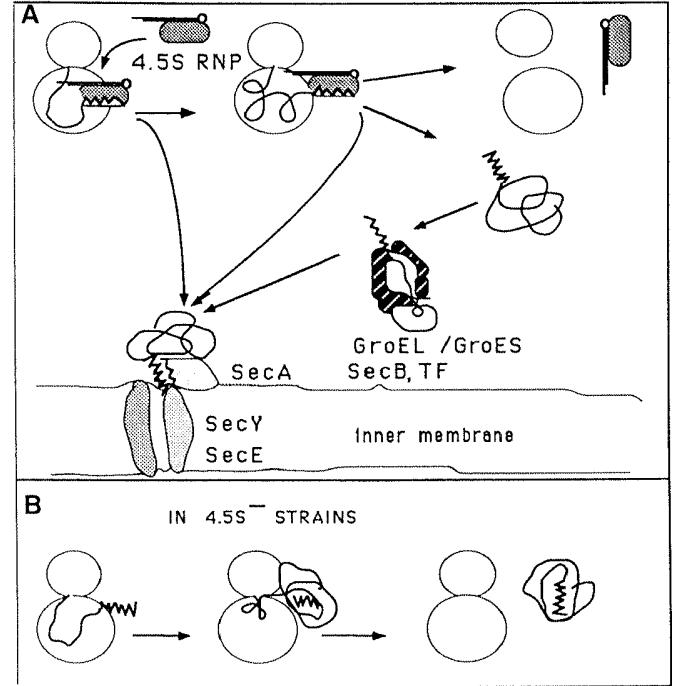

Figure 8. Hypothetical Model for the Function of 4.5S RNP in Protein Secretion

(A) 4.5S RNP interacts on the ribosome with the signal sequence of nascent pre- $\beta$-lactamase and possibly with the signal sequences of other preproteins. During translation the complex might interact with a membrane receptor, and translocation commences. Alternatively, the completed, translocation-competent protein might be released from the complex. If not immediately bound to the membrane receptor, the preprotein would associate with cytosolic chaperonins (SecB, GroEL/ GroES, trigger factor, or others), which would maintain its translocation competence until receptor binding.

(B) In the absence of 4.5S RNA, the hydrophobic signal sequence is folded within the protein, which cannot then be translocated.

related to SRP. Second, genetic and biochemical studies establish that the 4.5S/P48 RNP is likely to interact with a subset of nascent polypeptides on the ribosome. Third, P48 and SRP54 are clearly structurally homologous and are therefore likely to have related functions. SRP54 has been shown by cross-linking experiments to bind to the signal sequences of nascent peptides (Krieg et al., 1986; Kurzchalia et al., 1986). Last, the earliest effect of $4.5 \mathrm{~S}$ RNA depletion detected to date is the accumulation of pre$\beta$-lactamase, consistent with this being a primary defect.

From its homology with SRP and its established localization on the ribosome, we suggest that, at least in the case of pre- $\beta$-lactamase, $4.5 \mathrm{~S} / \mathrm{P} 48$ RNP interacts with the signal sequence of the nascent polypeptide in order to keep it in a translocation-competent conformation and available for interaction with other components of the secretory apparatus until release from the ribosome. To help relate the possible function of the 4.5S/P48 RNP to previously identified secretion factors, we present a hypothetical model in Figure 8.

Since there is no evidence that the 4.5S/P48 RNP possesses the other activities of SRP, translation arrest and membrane targetting, we envisage that following translation termination the complete preprotein is released into the cytosol. To maintain its translocation competence the preprotein would then interact with cytosolic factors until binding to the membrane receptor for translocation. Over- 
expression of the P48 component of the 4.5S/P48 RNP might impair growth and processing of pre- $\beta$-lactamase because it competes with the RNP for signal sequence or ribosome binding, but does not lead to a translocationcompetent preprotein

At present we have detected accumulation only of pre- $\beta$ lactamase; further analysis will determine whether the 4.5S/P48 RNP also plays a role in the secretion of other proteins. It remains possible that the primary defect in 4.5S RNA-depleted strains is in some other aspect of cell metabolism. However, because the lethality is suppressed by expression of SRP7S, it is likely that any such additional function is shared by eukaryotic SRP. What other aspect of metabolism might be affected is not clear. An obvious possibility would be translation; however, for reasons discussed above, a general role for $4,5 S / P 48$ in translation seems unlikely at present.

In the model presented here, we envisage that the 4.5S/P48 RNP is derived from an evolutionary ancestor of SRP, which possessed the crucial ability to bind the signal sequences of nascent polypeptides, but had not yet acquired the additional properties of SRP: translational inhibition and membrane targetting. Comparison of 4.5S RNA and SRP7S indicates that the latter has acquired distinct domains of RNA structure (Struck et al., 1988; Poritz et al., 1988b). It is therefore interesting to note that contemporary SRP still retains a structure composed of functionally distinct and separable domains (Siegel and Walter, 1988), which may reflect the evolutionary history of the particle.

\section{Experimental Procedures}

\section{Strains}

The effects of depletion of 4.5S RNA were tested in the strain FF283 (F-lac $\Delta \times 74$ ara D139 [araABOIC-leu] $\Delta 7679$ galu galK rpsLF' lac-pro, (acl $\left.{ }^{\circ}\right)$, a non-heat-inducible variant of strain FF164 described by Brown and Fournier (1984). In this strain, the endogenous ffs gene has been replaced with a kanamycin determinant by $\mathrm{P1}$ transduction, following introduction of the ffs gene under the control of a Tac promoter (TaC: $f f s$ ). The strain is kanamycin resistant and IPTG dependent. With continued growth, there is a loss of the $F^{\prime}$ episome at about $5 \%$ in liquid medium. Jm105 was chosen as a wild-type strain for the synthesis of 4.5S RNA. The other experiments were performed in strain MC4100.

\section{Plasmids}

Plasmids used for the construction of p7SH were pDR720 (Russell and Bennett, 1982), purchased from Pharmacia, and pKL915 (Stanley and Luzio, 1984), kindly provided by K. Stanley. Plasmids used for the in vitro binding and the overexpression of P48 were pSRP19 (Lingelbach et al., 1988): pSRP54-1 (Römisch et al., 1989); pDS12 (Bujard et al., 1987); pDS12-48, which is pDS12 containing the cloned P48 gene on a Sall-Hindill fragment from pBY39 (Byström and Bjork, personal communication) (which encodes the gene for the E. coli $48 \mathrm{~K}$ protein [P48] Byström et al., 1983); pET-8c-48, in which at the starting ATG of P48 a Ncol site was created by site-directed mutagenesis in M13mpt8 and the resulting Ncol-BamHI fragment was cloned into PET-8c (Studier et al., 1990)

\section{Media}

LB, M9, and M63 media were prepared according to Miller (1972). Antibiotics were used at a concentration of $100 \mu \mathrm{g} / \mathrm{ml}$ for ampicillin and 30 $\mu \mathrm{g} / \mathrm{ml}$ for kanamycin. Synthesis of 4.5S RNA was induced by replacing the glucose by lactose in the M9 medium or by adding $1 \mathrm{mM}$ IPTG. Synthesis of SRP7S RNA was induced by IAA ( $\beta$-indoleacrylic acid) at a concentration of $5 \mu \mathrm{g} / \mathrm{ml}$. MBP was induced by $0.2 \%$ maltose added in a glycerol M9 medium.

\section{In Vitro Binding}

Formation of an RNP particle was tested by modification of a previously published method (Lingelbach et al., 1988).

4.5S RNA (generously provided by M. Fournier, University of Massachusetts) was prepared from an overproducing $E$. coli strain harboring a multicopy plasmid with the ffs coding sequence fused to the $P 1$ and $P 2$ promoters of the rmb operon (J. Brosius and M. J. Fournier, unpublished data). Purification was by successive cycles of electrophoresis in native and denaturing gels of $10 \%$ polyacrylamide (Bourgaize et al., 1990).

In vitro transcripts from PSRP19 and P48 were translated for $30 \mathrm{~min}$ at $25^{\circ} \mathrm{C}$ in a wheat germ cell-free system. EDTA was added to a final concentration of $5 \mathrm{mM}$ to release nascent chains from ribosomes and IRNA, and incubation was continued for $15 \mathrm{~min}$. The translation reactions were then adjusted to $5 \mathrm{mM} \mathrm{MgOAC} 2$ and $500 \mathrm{mM}$ KOAc. Five microliters of SAP 54 or P48 or $10 \mu$ of SRP19 translation reaction and $1 \mu \mathrm{g}$ of 4.5S PNA or tRNA were mixed and incubated at $25^{\circ} \mathrm{C}$ for 30 $\mathrm{min}$. The volume was adjusted to $200 \mu \mathrm{l}$ with $500 \mathrm{mM} \mathrm{KOAc}, 5 \mathrm{mM}$ $\mathrm{MgOAC}_{2}, 50 \mathrm{mM}$ Tris ( $\mathrm{pH} 7.4$ ) (wash buffer). One-fifth of the reaction volume was removed and TCA precipitated to monitor total protein synthesis. Thirty microliters of DEAE-Sepharose CL-6B (Pharmacia) equilibrated in wash butfer was added to the remainder and incubated at $4^{\circ} \mathrm{C}$ for $15 \mathrm{~min}$. After centrifugation, the unbound supernatant fraction was TCA precipitated. The DEAE-Sepharose beads were washed twice with wash buffer, and DEAE-bound material was released at $4^{\circ} \mathrm{C}$ with $200 \mu \mathrm{l}$ of the same buffer adjusted to $2 \mathrm{M} \mathrm{KCl}$. Eluted protein was
TCA precipitated, and all samples were analyzed on $10 \%-15 \%$ Laemmli-type SDS-polyacrylamide gels.

\section{Sucrose Gradient}

Strains containing P7SH were grown in LB medium in the presence of IAA. Cultures were harvested at an $O_{600}$ of $0.8-1$, and cells were disrupted with glass beads (Sigma, G-1145) by vortexing for $5 \mathrm{~min}$ at $4^{\circ} \mathrm{C}$ in the $\mathrm{B} 100$ buffer $\left(100 \mathrm{mM} \mathrm{KCl}, 10 \mathrm{mM}\right.$ Tris- $\mathrm{HCl}\left[\mathrm{pH}\right.$ 7], $5 \mathrm{mM} \mathrm{MgCl}_{2}$, $0.01 \%$ Triton, $0.1 \mathrm{mM}$ PMSF, $1 \mathrm{mM} \mathrm{DTT}$ ). Half the lysate was treated with phenol at $4{ }^{\circ} \mathrm{C}$. Both native and phenol-extracted lysates were then loaded onto gradients of 5\%-20\% sucrose in B100 buffer. Gradients were centrifuged at $30,000 \mathrm{rpm}$ for $15 \mathrm{hr}$ in an SW40 rotor. Fractions of $500 \mu$ l were collected from the top, and the RNA was analyzed by Northern hybridization (Tollervey and Mattaj, 1987).

\section{Antibodies}

The polyclonal anti-SRP54/P48 antiserum 730 was raised in rabbit against a peptide derived from SRP54 (amino acids 103-117); this antiserum cross-reacts with the native and denatured $E$. coli $P 48$ protein. Cross-reactivity was verified by immunoprecipitating proteins from cells overexpressing the $\mathrm{P} 48$ protein and by Western blotting in the presence and absence of competition from the peptide against which the serum was raised.

\section{Western Blots}

One $O D_{600}$ unit of cells was taken at different points of the growth curves, spun down, heated in the Laemmli buffer for $5 \mathrm{~min}$ at $95^{\circ} \mathrm{C}$, and separated on $12 \%$ SDS-polyacrylamide gels. Proteins were electroblotted on an Immobilon membrane (Millipore) and visualized with antisera and ${ }^{35} \mathrm{~S}$-labeled protein A (Amersham). To specifically block the activity of serum 730 against $P 48$, competitor peptide $(1 \mu \mathrm{g} / \mathrm{ml})$ was added during incubation with the serum.

\section{Immunoprecipitation}

\section{Immunoprecipitation of RNPS}

Protein A-Sepharose was washed twice in B250 (same as B100 but $250 \mathrm{mM} \mathrm{KCl}$ ) and incubated with the antiserum in B150 (same as B100 but $150 \mathrm{mM} \mathrm{KCl}$ ) for $1 \mathrm{hr}$ at $4^{\circ} \mathrm{C}$, washed again, and shaken with a lysate prepared as for the sucrose gradient for $2-3 \mathrm{hr}$ at $4^{\circ} \mathrm{C}$. After centrifugation, the pellet was washed three times with B250, and the RNA was recovered (Tollervey and Mattaj, 1987). To specifically block the activity of serum 730 against P48, $1 \mu \mathrm{g}$ of the competitor peptide was preincubated with the protein A-Sepharose-bound antibody for 5 min prior to addition of the cell lysate.

\section{Immunoprecipitation from Strains Overproducing PA8}

For [ ${ }^{35}$ S]methionine labeling, cells were grown to an $\mathrm{OD}_{600}$ of 0.4 in LB, and P48 overexpression was induced with $1 \mathrm{mM}$ IPTG for the indi- 
cated times. Cells were labeled with $100 \mu \mathrm{Ci}$ of ${ }^{35} \mathrm{~S}$ ]methionine for 10 $\mathrm{min}$. Aliquots $(200 \mu \mathrm{l})$ were spun through $1 \mathrm{ml}$ of ice-cold wash buffer (10 mM Tris [pH 8.0], $1 \mathrm{mM}$ EDTA, $150 \mathrm{mM} \mathrm{NaCl}$ ), resuspended in 20 $\mu$ l of wash buffer containing $1 \%$ SDS, heated to $95^{\circ} \mathrm{C}$ for $3 \mathrm{~min}$, and diluted 1:20 with wash buffer containing 2\% Triton $X-100$. Immunoprecipitation of $\mathrm{P} 48$ was performed overnight, and precipitated complexes were analyzed by SDS-PAGE and fluorography.

\section{Northern Hybridization}

RNAs were extracted according to Tollervey and Mattaj (1987) and analyzed on $8.3 \mathrm{M}$ urea-8\% polyacrylamide gels. For Northern hybridization, gels were electroblotted onto a Hybond $\mathrm{N}$ membrane (Amersham). Probes were prepared from the Sall-Hindlll fragment of plasmid p801 (S. Brown and M. Fournier, unpublished data) for 4.5S RNA, from the EcoRI-Hindlll fragment of plasmid pTL30-1 (Ullu and Weiner, 1984) for human SRP7S, and from the Hindlll-Hindlll fragment of plasmid pcol2 (Ribes et al., 1988) for S. pombe SRP7S.

\section{Staining of E. coli Cells}

Cells were fixed in $2 \%$ paraformaldehyde in PBS at early exponential growth phase. Then the cells were dried down onto a glass slide and stained for $30 \mathrm{~s}$ in a solution containing $0.4 \%$ methylene blue chloride, $25 \%$ ethanol, and $0.007 \%$ potassium hydroxide. Excess stain was removed by several washes with PBS. Coverslips were mounted onto the cells in water, and the preparations were photographed while still wet.

\section{Acknowledgments}

We thank M. Wikström for plasmid pBY39, R. Gentz and A. Laminet for anti- $\beta$-lactamase antisera, R. Zimmermann for anti-OmpA serum, W. Boos for anti-MBP serum, and M. Müller for anti-AP serum. We are especially grateful to M. Fournier for the strain FF283, the purified 4.5S RNA and his advice. We also thank $H$. Gausepohl for synthesizing the peptide used for antibody production and M. Hentze, A. Lamond, I. Mattaj, and M. Beltrame for critical review of the manuscript.

The costs of publication of this article were defrayed in part by the payment of page charges. This article must therefore be hereby marked "advertisement" in accordance with 18 USC Section 1734 solely to indicate this fact.

Received February 5, 1990; revised August 2, 1990.

\section{References}

Bernstein, H. D., Poritz, M. A., Strub, K., Hoben, P. J., Brenner, S., and Walter, $P$. (1989). Model for signal sequence recognition from aminoacid sequence of $54 \mathrm{~K}$ subunit of signal recognition particle. Nature 340, 482-486.

Bochkareva, E. S., Lissin, N. M., and Girshovich, A. S. (1988). Transient association of newly synthesized unfolded proteins with the heatshock GroEL protein. Nature 336, 254-257.

Bourgaize, D. B., and Fournier, M. J. (1987). Initiation of translation is impaired in $E$. coli deficient in 4.5S RNA. Nature 325, 281-284.

Bourgaize, D. B., Phillips, T. A., Van Bogelen, R. A., Jones, P. G., Neidhardt, F. C., and Fournier, M. J. (1990). Loss of 4.5S RNA induces the heat shock response and lambda prophage in Escherichia coli. J. Bacteriol. 172, 1151-1154.

Brennwald, P., Liao, X., Holm, K., Porter, G., and Wise, J. A. (1988). Identification of an essential Schizosaccharomyces pombe RNA homologous to the $7 \mathrm{SL}$ component of signal recognition particle. Mol. Cell. Biol. 8, 1580-1590.

Brown, S. (1987). Mutations in the gene for EF-G reduce the requirement for 4.5S RNA in the growth of E. coli. Cell 49, 825-833.

Brown, S. (1989). Time of action of 4.5S RNA in Escherichia colit translation. J. Mol. Biol. 209, 79-90.

Brown, S., and Fournier, M. J. (1984). The 4.5S RNA gene of Escherichia coli is essential for cell growth. J. Cell Biol. 178, 533-550.

Brown, S., Thon, G., and Tolentino, E. (1989). Genetic selection and DNA sequences of 4.5S RNA homologs. J. Bacteriol. 171, 6517-6520. Bujard, H., Gentz, R., Lanzer, M., Stueber, D., Müller, M., Ibrahimi, I., Haeuptle, M. T., and Dobberstein, B. (1987). A T5 promoter-based transcription-translation system for the analysis of proteins in vitro and in vivo. Meth. Enzymol. 155, 416-433.

Byström, A. S., Hjalmarsson, K. J., Wikström, P. M., and Björk, G. R. (1983). The nucleotide sequence of an Escherichia coli operon containing genes for the $\operatorname{RNA}(\mathrm{m} 1 \mathrm{G})$ methyltransferase, the ribosomal proteins S16 and L19 and a 21-K polypeptide. EMBO J. 2, 899-905.

Campos, N., Palau, J., Torrent, M., and Ludevid, D. (1988). Signal recognition-like particles are present in maize. J. Biol. Chem. 263, 9646-9650.

Collier, D. N., Bankaitis, V. A., Weiss, J. B., and Bassford, P. J., Jr. (1988). The antifolding activity of SecB promotes the export of the $E$. coli maltose-binding protein. Cell 53, 273-283.

Crooke, E., Guthrie, B., Lecker, S., Lill, R., and Wickner, W. (1988). ProOmpA is stabilized for membrane translocation by either purified E. coli trigger factor or canine signal recognition particle. Cell 54, 1003-1011.

Fikes, J. D., and Bassford, P. J., Jr. (1989). Export of unprocessed precursor maltose-binding protein to the periplasm of Escherichia coli cells. J. Bacteriol. 169, 2352-2359.

Gardel, C., Benson, S., Hunt, J., Michaelis, S., and Beckwith, J. (1987). SecD, a new gene involved in protein export in Escherichia coli. J. Bacteriol. 169, 1286-1290.

Gilmore, R., Blobel, G., and Walter, P. (1982). Protein translocation across the endoplasmic reticulum. II. Isolation and chiaracterization of the signal recognition particle receptor. J. Cell Biol. 95, 470-477.

Goff, S. A., and Goldberg, A. L. (1985). Production of abnormal proteins in E. coli stimulates transcription of lon and other heat shock genes. Cell 41, 587-595.

Gundelfinger, E. D., Carlo, M. D., Zopf, D., and Melli, M. (1984). Structure and evolution of the 7SL RNA component of the signal recognition particle. EMBO J. 3, 2325-2332.

Hann, B. C., Poritz, M. A., and Walter, P. (1989). Saccharomyces cerevisiae and Schizosaccharomyces pombe contain a homologue to the $54 \mathrm{kD}$ subunit of the signal recognition particle that in $\mathrm{S}$. cerevisiae is essential for growth. J. Cell Biol. 109, 3223-3230.

Hsu, L. M., Zagorski, J., and Fournier, M. J. (1984). Cloning and sequence analysis of the Escherichia coli 4.5S RNA gene. J. Mol. Biol. 178, 509-531.

Kaine, B. P. (1990). Structure of the archaebacterial 7S RNA molecule. Mol. Gen. Genet. 221, 315-321.

Krieg, U. C., Walter, P., and Johnson, A. E. (1986). Photocrosslinking of the signal sequence of nascent preprolactin to the 54-kilodalton polypeptide of the signal recognition particle. Proc. Natl. Acad. Sci. USA $83,8604-8608$.

Kumamoto, C. A. (1989). Escherichia coli SecB protein associates with exported protein precursors in vivo. Proc. Natl. Acad. Sci. USA 86, 5320-5324.

Kumamoto, C. A., and Beckwith, J. (1983). Mutations in a new gene, Sec B, cause defective protein localization in Escherichia coli. J. Bacteriol. 154, 253-260.

Kumamoto, C. A., and Beckwith, J. (1985). Evidence for specificity at an early step in protein export in Escherichia coli. J. Bacteriol. 163, 267-274.

Kurzchalia, T. V. Wiedmann, M., Girshovich, A. S., Bochkareva, E. S. Bielka, H. and Rapoport, T. A. (1986). The signal sequence of nascent preprolactin interacts with the $54 \mathrm{~K}$ polypeptide of the signal recognition particle. Nature 320,634-636.

Kusukawa, N., Yura, T., Ueguchi, C., Akiyama, Y., and Ito, K. (1989). Effects of mutations in heat-shock genes groES and groEL on protein export in Escherichia coli. EMBO J. 8, 3517-3521.

Lee, C., and Beckwith, J. (1986). Cotranslational and posttranslational protein translocation in procaryotic systems. Annu. Rev. Cell Biol. 2, 315-336.

Lingelbach, K., Zwieb, C., Webb, J. R., Marshallsay, C., Hoben, P. J., Walter, P., and Dobberstein, B. (1988). Isolation and characterization of a cDNA clone encoding the $19 \mathrm{kDa}$ protein of signal recognition particle (SRP): expression and binding to 7SL RNA. Nucl. Acids Res. 16, 9431-9442. 
Meyer, D. 1.(1988). Preprotein contormation: the year's major theme in translocation studies. Trends Biochem. Sci. 13, 471-474.

Meyer, D. 1. Krause, E., and Dobberstein, B. (1982). Secretory protein translocation across membranes - the role of "docking protein." Nature $297,647-650$

Miller, J. H. (1972). Experiments in Molecular Genetics (Cold Spring Harbor, New York: Cold Spring Harbor Laboratory)

Moritz. A. and Goebel, W. (1985). Characterization of the 7S RNA and its gene from halobacteria. Nucl. Acids Fes. 13,6969-6979.

Oliver, D. B. and Beckwith, J. (1981). E. coll mutant pleiotropically defective in the export of secreted proteins. Cenl 25, 765-772.

Poritz. M. A., Siegel, V., Hansen, W., and Watter, P. (1988a). Small ribonucleoproteins in Schizosaccharomyces pombe and Yarrowia lipolytica homologous to signal recognition particle. Proc. Natl. Acad. Sci. USA 85. 4315-4319

Poritz, M. A., Strub, K., and Walter, P. (1988b). Human SAP RNA and E. coli 4.5S RNA contain a highly homologous structural domain. Cell $55,4-6$.

Pugsley, A. (1988). Protein secretion across the outer membrane of Gram-negative bacteria. In Protein Transfer and Organelle Biogenesis, Rathindra C. Das and Phillips W. Robbins, eds. (New York: Academic Press), pp. 607-642.

Randall, L. L., Hardy, S. J. S., and Tom, J. R. (1987). Export of protein: a biochemical view. Annu. Rev. Microbiol. 41, 507-541

Ribes, V., Dehoux, P., and Tollervey, D. (1988). 7SL. RNA from Schizosaccharomyces pombe is encoded by a single essential gene. EMBO J. 7, 231-237

Riggs, P. D., Derman, A. 1, and Beckwith, J. (1988). A mutation affecting the regulation of a $\sec A-\operatorname{lac} Z$ fusion defines a new sec gene. Genetics 118, 571-579.

Pörnisch, K. Webb, J. Herz, J., Prehn, S., Frank, R., Vingron, M., and Dobberstein, B. (1989). Homology of $54 \mathrm{~K}$ protein of signal-recognition particle, docking protein and two $E$. coll proteins with putative GTP. binding domains. Nature 340, 478-482

Russell, D. R., and Bennett, G. N. (1982). Construction and analysis of in vivo activity of $E$. coli promoter hybrids and promoter mutants that alter the -35 to -10 spacing. Gene 20, 231-243.

Sanz, P., and Meyer, D. I. (1988). Signal recognition particle (SRP) stabilizes the transtocation-competent conformation of presecretory proteins. EMBO J. 11, 3553-3557.

Schatz, P. J., Riggs, P. D., Jacq, A., Fath, M. J., and Beckwith, J. (1989). The secE gene encodes an integral membrane protein required for protein export in Escherichia coli. Genes Dev. 3, 1035-1044

Shiba, K. Ito, K., and Yura, T. (1984). Mutation that suppresses the protein export defect of the secY mutation and causes cold-sensitive growth of Escherichia coli. J. Bacteriol. 160, 696-701.

Siegel, V., and Walter, P. (1988). Functional dissection of the signal recognition particle. Trends Biochem. Sci. 13, 314-315

Stader, J., Gansheroff, L. J., and Silhavy, T. J. (1989). New suppressors of signal-sequence mutations, prIG, are linked tightly to the secE gene of Escherichia coli. Genes Dev. 3, 1045-1052.

Stanley, K. K., and Luzio, J. P. (1984). Construction of a new family of high efficiency bacterial expression vectors: identification of CDNA clones coding for human liver proteins. EMBO J. 3, 1429-1434.

Struck, J. C. R., Toschka, H. Y., Specht, T., and Erdmann, V. A. (1988) Common structural features between eukaryotic 7SL RNAs, eubacterial 4.5S RNA and SCRNA and archaebacterial 7S RNA. Nucl. Acids Res. 16, 7740 .

Struck, J. C. R., Lempicki, R. A., Toschka, H. Y., Erdmann, V. A., and Fournier, M. J. (1990). E. coli 4.5S RNA function can be complemented by heterologous bacterial RNA genes. J. Bacteriol. 172, 1284-1288. Studier, F. W., Rosenberg. A. H., and Dunn, J. J. (1990). Use of T7 RNA polymerase to direct the expression of cloned genes. Meth. Enzymol. $185,60-89$

Sugimoto, K., Sugisaki, H., Okamoto, T., and Takanami, M. (1977). Studies on bacteriophage fd DNA. IV. The sequence of messenger RNA for the major coat protein gene. J. Mol. Biol. 110, 487-507.
Tollervey, D., and Mattaj, I. (1987). Fungal small nuclear ribonucleoproteins share properties with plant and vertebrate U-snRNPS. EMBO J. $6,469-476$

Ullu, E. and Weiner, A. M. (1984). Human genes and pseudogenes for the 7SL RNA component of signal recognition particle. EMBO J. 3 , $3303-3310$

von Heijne, G. (1985). Structural and thermodynamic aspects of the transfer of proteins into and across membranes. Curr. Topics Membr Trans. 24, 151-179.

Walter, P., and Blobel, G. (1982). 7SL small cytoplasmic RNA is an integral component of the signal recognition particle. Nature 299, 691-698. Walter, P., and Blobel, G. (1983). Signal recognition particle: a ribonucleoprotein required for cotranslational translocation of proteins, isolation and properties. Meth. Enzymol. 96, 682-691.

Walter, P. and Lingappa, V. R. (1986). Mechanism of protein translocation across the endoplasmic reticulum membrane. Annu. Rev. Cell Biol. 2, 499-516.

Watanabe, M., and Blobel, G. (1989). SecB functions as a cytosolic signal recognition factor for protein export in $E$. coli. Cell 58, 695-705. Zwieb, C. (1985). The secondary structure of the 7SL RNA in the recognition particle: functional applications. Nucl. Acids Res. 13, 6101-6124. Zwieb, C., and Ullu, E. (1986). Identification of dynamic sequences in the central domain of 7SL. RNA. Nucl. Acids Res. 14, 4639-4657.

\section{Note Added in Proof}

The present address of Véronique Ribes is Institut Pasteur, 75724 Paris CEDEX 15, France. 